\title{
Demographics of cattle positive for Mycobacterium avium subspecies paratuberculosis by faecal culture, from submissions to the Cork Regional Veterinary Laboratory
}

Richardson EKB ${ }^{1}$, Mee $\mathrm{JF}^{1}$, Sánchez-Miguel $\mathrm{C}^{2}$, Crilly $\mathrm{J}^{1}$ and More SJ ${ }^{3}$

${ }^{1}$ Moorepark Dairy Production Research Centre, Teagasc, Fermoy, Co. Cork, Ireland

${ }^{2}$ Cork Regional Veterinary Laboratory, Model Farm Road, Bishopstown, Co. Cork, Ireland

${ }^{3}$ CVERA, University College Dublin, Dublin 4, Ireland

${ }^{4}$ Centre for Veterinary Epidemiology and Risk Analysis, UCD School of Agriculture, Food Science and Veterinary Medicine, University College Dublin, Belfield, Dublin 4, Ireland

\section{ABSTRACT}

The demography of bovine infections caused by Mycobacterium avium subspecies paratuberculosis (MAP) in Ireland is poorly defined. The objective of this study was to describe the demographics of cattle positive to MAP on faecal culture, based on submissions to the Cork Regional Veterinary Laboratory (Cork RVL) from 1994 to 2006. The study focused on all available faecal samples from adult cattle with non-responsive chronic diarrhoea that were submitted by private veterinary practitioners to Cork RVL for MAP culture. For each MAP-positive by faecal culture animal, data were collated from Cork RVL and Cattle Movement Monitoring Scheme (CMMS) records. Johne's disease (JD) was confirmed in 110 animals from 86 herds by the Cork RVL between 1994 and 2006, with a rate of positive cases between $15 \%$ and $18 \%$ over last four years of the study. Two breeds (Holstein/Friesian or Limousin) made up $78 \%$ of submissions. Movements were assessed for the 57 study animals with available movement information, $90 \%$ died within one year of the test and $26 \%$ tested positive in the herd they were born into. The study provides preliminary information about movement trends and demographics of animals with MAP positive submissions. Although the study area is restricted, it includes the most intensive (and economically-important) dairy region in Ireland. The demographics of JD infection from the study area are in agreement with international reports. Further work is required to determine demographic trends, incidence and prevalence of JD throughout Ireland. It is hoped this work may contribute to the development of a surveillance strategy for MAP by regional veterinary laboratories.

KEYWORDS: cattle demographics, cattle movements, faecal culture, Johne's disease, laboratory submissions, network diagram, retrospective data

\section{CORRESPONDING AUTHOR:}

Esther Richardson

E-mail: richardson.esther@gmail.com

Demography of bovine infections caused by Mycobacterium avium subspecies paratuberculosis (MAP) is poorly defined in Ireland. Disease caused by MAP infection is commonly referred to as Johne's disease (JD) which can affect farm performance through reduced milk production and increased culling (Ott et al. 1999; Lombard et al. 2005). Potential links 
between JD in cattle and Crohn's disease in humans could lead to international barriers to trade in milk and milk products for countries with poor surveillance and control of the disease which has caused concern in Ireland (Stabel 2000; O'Reilly et al. 2004). Between 1932 and 1992, 92 MAP cases were reported sporadically in Ireland, primarily in imported animals (Barrett et al. 2006). Following the removal of pre-importation test certification and post-importation quarantine as a consequence of the introduction of the single European market in 1992, there was a dramatic increase in the number of cattle imported from countries where MAP is endemic within the EU. Between 1992 and 2004, 85,000 cattle were imported into Ireland from continental Europe (Barrett et al. 2006). In comparison, between 1979 and 1990 only 1,194 potential breeding animals were imported from these countries (Trade Statistics, Central Statistics Office, Ireland 2008). Results from Irish surveys by O'Doherty et al. (2002) identified MAP in $36 \%$ of the 36 surveyed herds in 1997, and Barrett et al. (2006) reported 232 MAP infected animals in 106 surveyed herds between 1995 and 2002. These results are in agreement with international studies (Hayton 2007; Khol et al. 2007) which suggest that the prevalence of paratuberculosis is increasing in a number of countries and that earlier warnings have not been taken seriously enough (Chiodini et al. 1984). Despite this, there have been no studies published on the temporal trends of MAP infections in Irish herds.

Regional veterinary laboratories (RVLs) of the Department of Agriculture, Fisheries and Food (DAFF) are used in Ireland to analyse samples for the presence of MAP. Samples with accompanying animal and herd details are submitted by private veterinary practitioners to their local RVL for testing. The Cork RVL is located in and services the most intensive dairying region of Ireland, Munster, from which $61 \%$ of the national dairy calves originated in 2006 (CMMS 2006).

Faecal culture is accepted internationally as the confirmatory test for detection of MAP in cattle (Collins 2003). The test sensitivity increases with the rate of bacterial shedding of the animal, whereas specificity is generally considered to be $100 \%$ (Whitlock et al. 2000).

Numerous risk factors have been identified which are associated with the likelihood of an animal testing positive to MAP. One of the most important is age: infected animals are most likely to test positive from 2.5 to 5.5 years (Nielsen and Ersboll 2006) or from third lactation onwards (Tavornpanich et al. 2006), due to the chronic nature of JD. Secondly, cattle breed may be a factor. In a Belgium study using a commercial serum ELISA test, dairy animals were found more likely to test positive than beef animals (Boelaert et al. 2000). While no genetic susceptibility has been conclusively demonstrated within dairy breeds, Jersey cattle have been found more likely to test positive than Holstein/Friesian or other 'larger' dairy breeds (Cetinkaya et al. 1997; Jakobsen et al. 2000). Comparative studies on such risk factors in Irish cattle have not been published.

The objective of this study was to describe the demographics of cattle positive to MAP on faecal culture, based on submissions to the Cork Regional Veterinary Laboratory from 1994 till 2006.

\section{MATERIALS AND METHODS General}

A retrospective study was conducted on laboratory data collected by the Cork Regional Veterinary Laboratory (Cork RVL) from 1994 to 2006. The study was purely descriptive and did not involve demographic comparisons between animals with and without MAP.

The study included all faecal samples from adult cattle with non-responsive chronic diarrhoea that were submitted by private veterinary practitioners to Cork RVL for MAP testing from 1994 (when feacal culture testing for JD began to be established in Ireland) till 2006. The Cork RVL, located in Cork city, County Cork, Ireland, provides a diagnostic service to private veterinary practitioners in County Cork, and some parts of the bordering counties of Clare, Kerry, Waterford, Limerick and South Tipperary. Throughout the study period, submitted faecal samples were cultured for MAP using Herrold's egg yolk agar (HEY) as described previously (Egan et al. 1999). MAP was considered to be present if the culture yielded acid-fast bacilli that required mycobactin $\mathrm{J}$ for growth and took at least five weeks to grow. There was no retesting of faecal positive animals. Each MAP-positive animal was subsequently termed a 'study animal'.

\section{Data collection and management}

For each study animal, data were collated from Cork RVL and Cattle Movement Monitoring Scheme (CMMS) records and managed using Microsoft Excel (Microsoft Corporation, Redmond, WA, USA). The CMMS system, which is managed by DAFF, captures all movements of cattle within Ireland and has been in operation since January 1, 2000, although dates of birth were not recorded for pre-1996 born animals. The following CMMS data were used: tag number, date of birth, breed, animal type, exit date (date of death), life status, dam tag number, the date and type of movement, and the origin and destination of movement. Animal type referred to age and gender categories including bull (un-castrated male), bullock (castrated male), cow (female post-parturition), and heifer (female pre-parturition). Animal 'life status' was categorised as dead, alive, unknown or unconfirmed dead (where animals were suspected dead, but death was not confirmed in the system, as may occur when the animal has died on-farm). Type of movement was private sale, through a mart, to a knackery, factory or an on-farm death. The origin and destination of movement was a herd, mart, abattoir (referred to in Ireland as a 'factory'), knackery or unknown. The following Cork RVL data were used, when available: laboratory reference number, test date (the date the sample submission was received by the Cork RVL), type of test and species tested, herd location and general comments (including age, breed, and animal tag number, as well as other background information, such as whether the animal had been imported). Animals coded as either 'Holstein' or 'Friesian' were grouped together as 'Holstein/Friesian' in this study. 


\section{Data analysis}

Analyses were conducted using Excel with three different subsets of the data: $A, B$ and $C$. The unit of interest was the submission in part A or the animal in parts $B$ and $C$. Each section had a differing number of submissions or animals available for analysis, as follows:

Part A: All faecal submissions from cattle from 1994 to 2006 for which MAP culture was requested

Descriptive analyses were conducted to determine the total number of submissions and the number positive on MAP culture, in total and by year.

Part B: The study animals (all animals positive on faecal culture) from 1994 to 2006

The location of herds in which all study animals were located, and of the Cork RVL, was mapped using ArcVIEW 9.1 (ERSI, Redlands, CA, USA). To ensure farm anonymity, the location of each herd was represented at the centroid of the relevant district electoral division. Descriptive analyses were conducted to describe the percentage of imported study animals, number of study animals per farm, and the distribution of study animals by animal type, breed and year. An imported animal was defined as an animal that was recorded as imported by the laboratory records or had a non-Irish Tag Number.

Part C: All study animals for which CMMS data were available

Descriptive analyses were conducted to determine the 'current' life status of each animal as on July 5, 2007, age at test and time from sample submission to death (for animals that died). Several methods were used to assess movement records. Movement data were summarised (average number of movements per animal) prior to and following test submission date. A social network diagram was developed using Ucinet software (Borgatti et al. 2002) to represent lifetime movement patterns. Network analysis is used to explore the characteristics of subgroups and individuals that make up a network. Animals with no recorded date of birth or source herd had date of birth or herd recorded as 'Unknown'. The number and longevity of recorded offspring from all female animals was

Figure 1: Number of submissions (Part A), animals (Parts B and C), and numbers of submissions/animals available within each part of analyses.

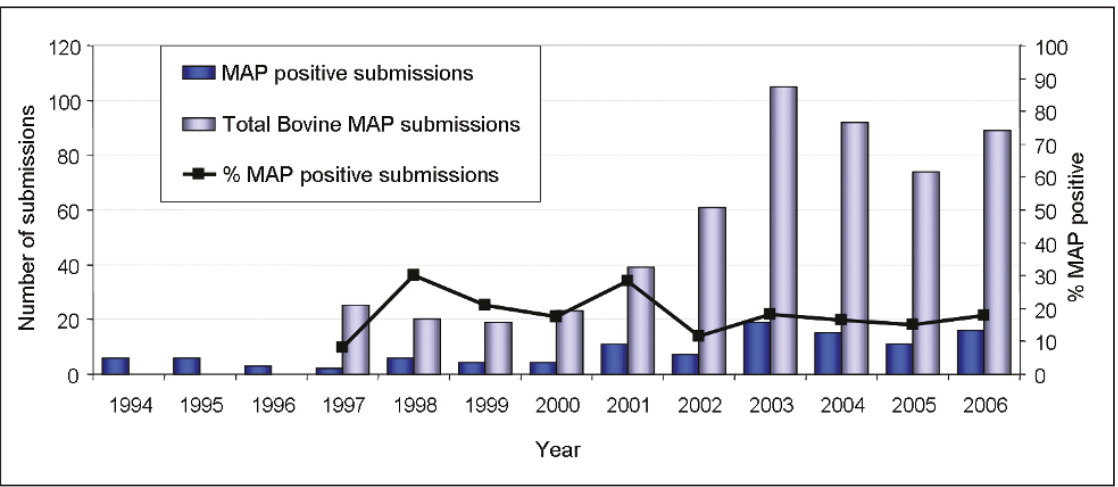

Figure 2: The total number of submissions to the Cork RVL for MAP faecal culture, and the number and percentage of submissions that were MAP-positive, by year between 1994 to 2006. Percentage positive from total are given from 1997 to 2006. determined, as was the life status of these offspring as on July 5, 2007.

\section{RESULTS}

Part A: Submissions to Cork RVL for MAP faecal culture (1994-2006)

At the Cork RVL, reliable records on the total number of submissions for MAP culture and the number of MAPpositive submissions were available from 1997 to 2006 and 1994 to 2006, respectively. From 1997 to 2006, 547 faecal samples were submitted to Cork RVL for MAP faecal culture (Figure 1). Total numbers of faecal samples submitted each year for testing has steadily increased 
(Figure 2). From 1994 to 2006, 110 MAP-positive samples were detected (Figure 1): the first in 1994. The percentage of submissions per year that were MAP-positive from 1997 until 2006 and fluctuated between $8 \%$ and $30 \%$ with a mean annual percentage of $18 \%$. Less variation occurred from 2003 through to 2006 where the mean annual percentage of positive submissions was $17 \%$ with a range of 15-18\% (Figure 2).

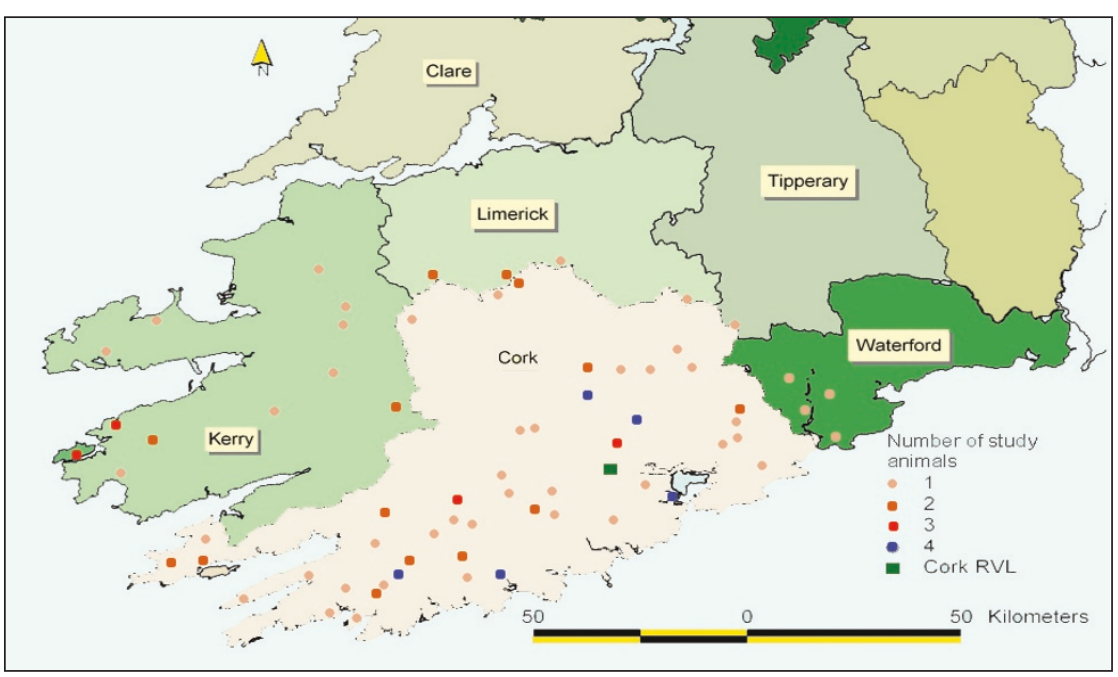

Figure 3: The location of the Cork Regional Veterinary Laboratory and of the $\mathbf{1 0 9}$ animals that were positive to MAP on faecal culture during 1994 to 2006. To ensure farm anonymity, the location of each study animal was represented at the centroid of the relevant district electoral division. The size of the circle reflects the number of study animals in each district electoral division.

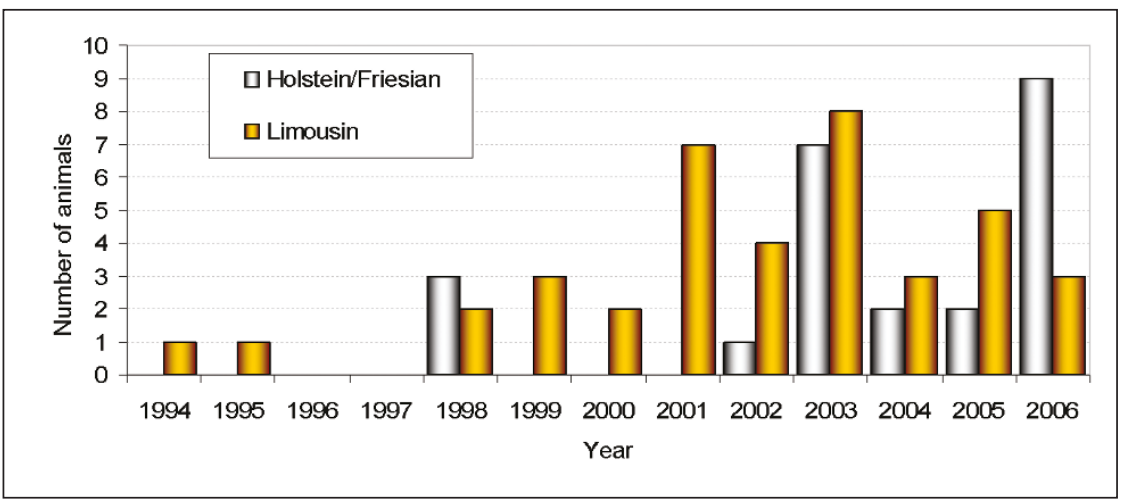

Figure 4: The number of Holstein/Friesian and Limousin animals that were confirmed MAP-positive on faecal culture at Cork Regional Veterinary Laboratory, by year.

Table 1: Distribution of 82 study animals (MAP positive on faecal culture) by breed. Animals coded as either 'Holstein' or 'Friesian' were referred to as 'Holstein/Friesian' in this study. Breed data were missing for 28 study animals

\begin{tabular}{|l|l|l|l|l|l|}
\hline & \multicolumn{2}{|c|}{$\begin{array}{c}\text { MAP-positive } \\
\text { submissions }\end{array}$} & & \multicolumn{2}{|c|}{$\begin{array}{l}\text { MAP-positive } \\
\text { submissions }\end{array}$} \\
\hline Breed & Count & $\%$ & Breed & Count & $\%$ \\
\hline (AU) Aubrac & 2 & $2 \%$ & (JEX) Jersey cross & 1 & $1 \%$ \\
\hline (CH) Charolais & 3 & $4 \%$ & (LM) Limousin & 40 & $49 \%$ \\
\hline (CHX) Charolais cross & 1 & $1 \%$ & (LMX) Limousin cross & 4 & $5 \%$ \\
\hline (HF) Holstein/Friesian & 24 & $29 \%$ & (NOX) Normande cross & 1 & $1 \%$ \\
\hline (JE) Jersey & 4 & $5 \%$ & (SI) Simmental & 2 & $2 \%$ \\
\hline
\end{tabular}

Part B: Study animals (animals MAP positive on faecal culture) from 1994 to 2006 and of the Cork RVL, is presented in Figure 3. Thirteen (15\% of the 86$)$ herds were reported with more than one study animal: eight of these herds had multiple positive

submissions (from two to four animals) taken from the herd within the same year, five had multiple positive submissions (from two to four animals) from the herd over a period of two to five years. Of 104 study animals with type recorded, 34\% were bulls, 1\% bullocks, 61\% cows and 4\% heifers. Table 1 shows a breakdown of study animals by breed. Of 82 animals with breed recorded, 64 (78\%) animals were Holstein/Friesian or Limousin. A further four animals were Limousin cross, whereas no Holstein/Friesian cross animals were reported. The number of Limousin and Holstein/Friesian study animals by year is presented in Figure 4. The first recorded confirmed case of JD at Cork RVL (in 1994) was a Limousin cow, and there has been a regular detection of MAP-positive Limousin animals subsequently. In contrast, reported MAP-positive submissions from Holstein/Friesian cows have been more sporadic, and appeared to increase in number over time. Among the MAP-positive Limousins, 62.5\% (25/40 Limousins) were bulls and among the MAP-positive Holstein/Friesians, 95.8\% (23/24 Holstein/Friesians) were cows.

\section{Part C: Study animals for which CMMS} records were available

From the 110 study animals, there were 57 for which CMMS records were available (Figure 1). At the time of analysis (July 5, 2007), 50 (87.7\%) of these animals were dead, three (5.3\%) were alive, one (1.8\%) had an unknown status, and three (5.3\%) were unconfirmed dead. The average age at test was four years ( $\mathrm{SD}=1.6$ years). As an estimate of survival, within 50 and 100 days of the submission date, $52 \%$ and $70 \%$ of study animals, respectively, had died or were slaughtered. Within 12 and 18 months of the sample submission date, $90 \%$ and $98 \%$ of study animals, respectively, had died or 
were slaughtered. One Holstein/Friesian cow survived for four years post submission date.

Movement data were available from 1999 to 2007 for 54 study animals (Figure 1). From this data 118 movements were recorded (92 were 'live' movements and 26 'dead': the latter refers to the movement of dead animals directly to knackeries from herds). Most animals had two movements recorded over their lifetime, one movement before, and one movement after the test was submitted to the lab. There were 14 animals (26\%) diagnosed with MAP in their herd of origin (source herd), whereas $74 \%$ tested positive in secondary or later herds. Movement count data was skewed to the right due to one animal having a total of nine movements, eight prior to testing, and one after. In total, $48 \%$ of animals with movement data were sent to knackeries which mean these study animals all died on-farm.

The pattern of lifetime movements for the 54 animals is presented in Figure 5. In network analysis a component is each group of connected nodes (data points). The 'main' component of a social network is much larger than the next largest component (Network Analysis Course 2006), and in this analysis a main component was identified, linking 39 of the study animals and included $91 \%$ of recorded animal movements in the data set. Limousin animals were the predominant breed in the main component of the social network. The remaining 15 animals not in the main component were linked either through smaller components (two groups of three linked animals, two groups of two linked animals) or did not have any movements in common with other study animals (five animals). In Figure 5, three reference groups which highlight movements of interest in the network are outlined; the outward movement of multiple positive animals from a single source herd (A), the movement of positive animals from the source herd into a subsequent herd where they tested positive and then moved to a factory or knackery (B: this pattern was frequently observed), and imported animals entering the network of Irish herds before testing positive (C: this was observed on five occasions in the network as the remaining seven imported animals either did not have tags reported, or traceable tags). Many of the study animals were connected through outward movements from two source herds and through inward movements to two knackeries and a factory. Few animals were diagnosed at their herd of origin.

Data were available on 117 offspring from 33 of the study animals (an average of 3.5 offspring per dam), including 27 $(23 \%)$ that were alive on July $5,2007$. In total, 40 female and 16 male offspring from culture positive dams lived for two years or more.

\section{DISCUSSION}

This paper describes the demographics of cattle positive to MAP on faecal culture, based on submissions to the Cork Regional Veterinary Laboratory during 1994 to 2006. The study has provided a valuable insight into aspects of JD epidemiology in dairy and beef populations in the south and southwest of Ireland.

In the Cork RVL, JD was first diagnosed by culture in 1994, two years after Irish importation policies were changed for cattle from the EU. This is coincident with the widespread view that these international policy changes adversely affected the JD status of Irish cattle herds (O'Reilly et al. 2004). There is evidence in this study that imported animals have played a role in JD epidemiology in Ireland. Among the study animals $10 \%$ originated from outside of Ireland. This suggests that imported animals were highly over represented in the study 
considering the maximum possible percentage of imports present in the national cattle herd in 2004 was $1.3 \%$ (CMMS report 2004; Trade Statistics, Central Statistics Office, Ireland; personal communication 2008). This figure is likely to be lower given deaths in imported animals. This over representation may be due to veterinary treatment being more readily given to imported stock, but also could indicate a higher incidence of JD in this group.

This study found that $15 \%$ of herds had multiple submissions and $91 \%$ of study animals with movement data had movements in common with other study animals. These findings suggest both spatial and temporal clustering, as would be expected given that JD is considered to be readily spread to susceptible animals (Nielsen et al. 2002). The average age of test-positive animals was four years (SD 1.6 years), which is a similar age to that observed in previous JD studies (Chiodini et al. 1984; Nielsen and Ersboll 2006). The majority of reported study animals were either Holstein/Friesian cows or Limousin bulls. These breeds are economically significant in Ireland, and have been associated with the disease in the past, both in Ireland and abroad (Pavlik et al. 2000; Barrett et al. 2006). Dairying predominates in the study area, hence the large number of Holstein/Friesian cows represented. Similarly, the 2006 CMMS Report identifies the general popularity of the Limousin breed in Ireland. Nationally, Limousins were the third most recorded sire breed after Friesian and Charolais in 2005, where 19.1\% of 2.15 million calves born in 2005 were to Limousin sires, and $5.6 \%$ of all calves born were pure bred Limousin calves (CMMS report 2006). However, as the study area was not predominantly a beef farming region the high number of Limousins (particularly bulls) among the study animals may reflect a higher JD prevalence in this breed, possibly due to a high level of movement between limousin herds as suggested by the network study or a higher breed susceptibility. Susceptibility of a breed (i.e., Jersey) to JD could be an issue for importing and promoting certain breeds in herds that already have reported cases of JD infection on the farm (Cetinkaya et al. 1997; Jakobsen et al. 2000). Alternatively, it may highlight the high level of awareness and reporting of this disease among Limousin breeders. This was recognised in the network diagram as a cluster of submissions where a Limousin cattle breeder, on identifying the disease in his stock, contacted his clients and tested animals that had been previously sold. Furthermore, initiatives have been taken within the Limousin breed society towards JD control, including changing breed society regulations to require testing for JD in conjunction with other requirements before an animal can be sold at a breed society sale.

JD incidence among submitted samples remained steady at approximately $17 \%$ per annum over the last four years of the study. This is certain to be an underestimate because bacterial shedding is intermittent and test sensitivity in less than 100\% (Whitlock et al. 2000). Increasing submission rates over the time period were noted. There are several possible explanations for this, including: increased demand for laboratory confirmation of diagnosis by private veterinarians in the area; increased JD awareness in veterinary practitioners and/or farmers in the area; and, increasing numbers of animals in the catchments area showing clinical signs of JD or with non-responsive chronic diarrhoea.

Results from the network analysis (Figure 5) highlight a range of issues relevant to the spread of infection on farms in this study. There was a high level of connectivity between study animals, consistent with the role of animal movement in the spread of MAP infection (Benedictus et al. 2000). Most animals (74\%) were not detected in their source herd, but tested positive in later herds. This may have contributed to the spread of infection to 64 herds. As highlighted in the network analysis, there were two examples of study animals from a single infected herd spreading to multiple herds. In each case, the study animals were Limousin bulls. Such spread from a single herd of infected animals that are likely to be retained on the farm for some time (either to breed from or fatten) highlights a potential pool of JD for herds sourcing untested bulls. Prior to this study, it was generally accepted that JD was introduced into dairy herds by beef bulls however, there were few links between beef and dairy herds in the network which raises questions about the source of infection in dairy herds. It should be noted that most study animals had few movements over their lifetime (generally one before and one after their test date), and therefore spread via cattle movements is likely to be slow, but incremental as few new herds would be exposed per infected study animal. Following diagnosis (faecal culture positive), it is likely that most study animals were highly infectious during the subsequent period prior to culling. It is of concern that one study animal was retained for some years following diagnosis.

It is of concern that there was no apparent follow-up of JD infection in the offspring of study animals. Offspring of confirmed MAP infected animals represent a potential source of infection if retained or sold to other farms. Research has shown that vertical cow-calf transmission of JD readily occurs (Stabel 2000; Weber 2006) as does horizontal calf-calf transmission thought to occur when MAP 'passes though' the gut of infected calves in the first few weeks post infection, thus causing the newly-infected calf to in turn be infectious to other co-habiting young calves (van Roermund et al. 2007). This highlights the infection risk associated with the offspring of MAP-positive dams. In this study, $34 \%$ of female offspring lived longer than two years, suggesting that many may have had an opportunity to spread infection within their home premises. These results suggest a widespread lack of JD awareness and/ or concern. In total, 26 (48\%) of the Part C study animals died on farm. These situations represent a substantial loss of income (foregone income from sale, additional cost associated with carcase collection by knackeries) and a welfare issue.

There are several questions arising from this research that require further data to clarify. One approach to 
gathering this data and answer these questions is to develop a method of on-going surveillance in the area. There are substantial benefits from effective and efficient surveillance strategies for JD, both locally and nationally. With surveillance information, it would be possible to critically evaluate JD incidence in Ireland, as well as issues relating to transmission and spread. In this study, JD status data was not available on animals from the rest of the herd in which the study animal was identified, nor on animals in other herds the study animal had been in over its lifetime. Within-herd transmission risk from a study animal could be assessed if test results were obtained from their offspring or herd. This data would provide insights into the reproductive rate $\left(R_{0}\right.$, both within- and between-herds [Dietz 1993; Halloran 1998]) in Ireland, as well as a clearer understanding of potential pathways for spread between farms. As demonstrated in the current study, regional veterinary laboratories routinely generate data (so-called passive surveillance [Buehler 1998]) relevant to local and national surveillance. With active surveillance (that is, the collection of additional data or additional tests on routine samples [Buehler 1998]), it may be possible to extract further information of relevance to the questions at hand. Whole herd tests could be conducted during other regular herd activities, such as brucellosis sampling to clarify questions regarding spatial and temporal clustering of the disease. There are additional questions about the study animals for which data are currently lacking. For example, are there identifiable factors differentiating multiple submission herds from single submissions herds? Did single submission herds have further cases that were not reported to the lab? How reliably did Elisa identify study animals? What is the role of imported stock in native JD infections and spread in Ireland?

It is of concern that only 57 (52\%) study animals could be matched to CMMS data. For the remaining 53 study animals, $49 \%$ had no tag number reported to the Cork $\mathrm{RVL}$, and $51 \%$ had tag numbers that did not match to CMMS records, in some cases due to submission under a short farm-management tag number (i.e., jumbo tag). Currently, despite the fact that JD is a notifiable condition in Ireland, many farmers are very reluctant to report the presence of JD in their animals, either to other farmers or to DAFF. Submission and testing of 'anonymous' animals which cannot be accurately individually identified is likely a reflection of this concern. There is a real need for policy makers to address this concern as it substantially curtails the ability of the RVL to follow-up cases and of government and industry to proactively address the issue of JD in Irish cattle herds.

There are a number of limitations to this study, which need to be considered during study interpretation. This study reported on positive faecal culture results which are more suited to testing the later stages of the disease (Whitlock et al. 2000). A range of other results were available from Cork RVL MAP testing (ELISA results, and results from other methods of detection such as Ziehl-Nielsen), which were not considered in this study. Thus, the study does not account for many animals positive by MAP ELISA submission to the lab during the study period. We elected to focus solely on faecal culture positive animals to clearly define the JD status of study animals by avoiding diagnostic issues over ELISA test sensitivity ( 40.8\%) and specificity ( 99.8\%) changing with the number of tests on an animal, or using ELISA positive animals that were culture negative (Whitlock et al. 2000; Dufour 2004). Although movement patterns may have changed over time, movement data were not routinely available prior to 1999 . By definition, the RVL submissions were opportunistic, and as a consequence may not be representative of the broader reference population. A range of factors influence the decision of farmers and veterinarians to submit to RVLs, including attitudes and physical location, as well as their knowledge and personal interest in JD. Some data (for example, breed, type etc.) were not available, and as a result, some study animals were excluded from some analyses.

Network analysis is an established method used to describe and analyse complex movement data. This approach is of particular relevance to animal disease control, providing insights into a range of issues including investigating the basic reproductive number for an infectious disease (Christley et al. 2005), investigating patterns of animal movements during the 2001 foot-and-mouth disease outbreak (Ortiz-Pelaez et al. 2006) and predicting tuberculosis transmission patterns in possums (Corner et al. 2003). The method proved useful in this study for examining trends in the movement of animals over time. Johne's disease was regularly confirmed by the Cork RVL between 1994 and 2006, particularly so in the last four years of the study. The study provides preliminary information about trends in MAP submissions and animals with MAP positive submissions which are consistent with current international understanding of the disease in cattle herds. Although the study area is restricted, it includes the most intensive (and economically-important) dairy region of Ireland. Further work is required to determine the demographics, spread and prevalence of JD in Ireland.

\section{ACKNOWLEDGEMENTS}

The authors wish to thank Martin Hayes and Peter Maher at DAFF for their generous help in retrieving and providing CMMS data for the study. The authors would like to thank Guy McGrath from CVERA for mapping submission locations within the catchments area of the study. Rob Christley is to be acknowledged for his willingly assistance in providing his network analysis course notes, for with the authors are most grateful. The authors would like to acknowledge the financial support of CVERA in the writing of this paper.

\section{REFERENCES}

Barrett D, Good M, Hayes M et al. (2006) The economic impact of Johne's disease in an Irish dairy herd: A case study. Irish Veterinary Journal 59, 282-288.

Benedictus G, Verhoeff J, Schukken YH et al. (2000) Dutch paratuberculosis programme history, principles and development. Veterinary Microbiology 77, 399-413. 
Boelaert F, Walravens K, Biront P et al. (2000) Prevalence of paratuberculosis (Johne's disease) in the Belgian cattle population. Veterinary Microbiology 77, 269-281.

Borgatti SP, Everett MG and Freeman LC (2002) Ucinet for Windows: Software for Social Network Analysis. Harvard, MA: Analytic Technologies.

Buehler JW (1998) Surveillance. In: Modern Epidemiology. Rothman KJ, Greenland S (eds). Philadelphia, Lippincott-Raven Publishers. Pp 448-449.

Cetinkaya B, Erdogan HM and Morgan KL (1997) Relationships between the presence of Johne's disease and farm and management factors in dairy cattle in England. Preventive Veterinary Medicine 32, 253-266.

Chiodini RJ, van Kruiningen HJ and Merkal RS (1984) Ruminant paratuberculosis (Johne's disease): The current status and future prospects. The Cornell Veterinarian 74,218-262.

Christley RM, Pinchbeck GL, Bowers RG et al. (2005). Infection in social networks: using network analysis to identify high-risk individuals. American Journal of Epidemiology 162, 1024-1031.

Collins MT (2003) Update on paratuberculosis: 2. Pathology and diagnosis. Irish Veterinary Journal 56, 619-623.

CMMS (2004) Statistics report 2004. [Internet] The Department of Agriculture and Food, Dublin, Ireland. Available from: www.agriculture.gov.ie

CMMS (2006) Statistics report. [Internet] National Beef Assurance Division. The Department of Agriculture and Food. Dublin, Ireland. Available from: www.agriculture. gov.ie

Corner LAL, Pfeiffer DU and Morris RS (2003) Socialnetwork analysis of Mycobacterium bovis transmission among captive brushtail possums (Trichosurus vulpecula). Preventive Veterinary Medicine 59, 147167.

Dietz K (1993) The estimation of the basic reproduction number for infectious diseases. Statistical Methods in Medical Research 2, 23-41.

Dufour B, Pouillot R and Durand B (2004) A cost/benefit study of paratuberculosis certification in French cattle herds. Veterinary Research 35, 69-81.

Egan J, Weavers E and O'Grady D. (1999). An evaluation of diagnostic tests for Johne's disease in cattle. Irish Veterinary Journal 52, 86-89.

Halloran ME (1998) Concepts of infectious disease epidemiology. In: Modern Epidemiology. Pp 536542. Rothman KJ, Greenland S (eds). Philadelphia, Lippincott-Raven Publishers.

Hayton AJ (2007) Johne's disease. Cattle Practice 15, 79-87.

Jakobsen MB, Alban L and Nielsen SS (2000) A crosssectional study of paratuberculosis in 1155 Danish dairy cows. Preventive Veterinary Medicine 46, 15-27.

Khol JL, Damoser J, Dunser M et al. (2007)

Paratuberculosis, a notifiable disease in Austria-Current status, compulsory measures and first experiences. Preventive Veterinary Medicine 82, 302-307.
Lombard JE, Garry FB, McCluskey BJ et al. (2005) Risk of removal and effects on milk production associated with paratuberculosis status in dairy cows. Journal of the American Veterinary Medical Association 227, 19751981.

Network Analysis Workshop: Course Notes (2006) PostISVEE XI course. Contact: Christley RM, The University of Liverpool, Liverpool, United Kingdom.

Nielsen SS, Grohn RL, Quaas L et al. (2002) Paratubertulosis in Dairy Cattle: Variation of the Antibody response in offspring attributable to the dam. Journal of Dairy Science 85, 406-412.

Nielsen SS, Ersboll AK (2006) Age at occurrence of Mycobacterium avium subspecies paratuberculosis in naturally infected dairy cows. Journal of Dairy Science 89, 4557-4566.

Ortiz-Pelaez A, Pfeiffer DU, Soares-Magalhães RJ et al. (2006) Use of social network analysis to characterise the pattern of animal movements in the initial phases of the 2001 foot and mouth disease (FMD) epidemic in the UK. Preventive Veterinary Medicine 76, 40-55.

Ott SL, Wells SJ and Wagner BA (1999) Herd-level economic losses associated with Johne's disease on US dairy operations. Preventive Veterinary Medicine 40, 179-192.

O'Doherty A, O'Grady D, O'Farrell K et al. (2002) Survey of Johne's disease in imported animals in the Republic of Ireland. Veterinary Record 150, 634-636.

O'Reilly CE, O'Connor, L, Anderson W et al. (2004) Surveillance of bulk raw and commercially pasteurised cows' milk from approved Irish liquidmilk pasteurisation plants to determine the incidence of Mycobacterium paratuberculosis. Applied and Environmental Microbiology 70, 5138-5144.

Pavlik I, Matlova L, Bartl J et al. (2000) Parallel faecal and organ Mycobacterium avium subsp. paratuberculosis culture of different productivity types of cattle. Veterinary Microbiology 77, 309-324.

Stabel JR (2000) Johne's disease and milk: do consumers need to worry? Journal of Dairy Science 83, 16591663.

Tavornpanich S, Gardner IA, Carpenter TE et al. (2006) Evaluation of cost-effectiveness of targeted sampling methods for detection of Mycobacterium avium subsp paratuberculosis infection in dairy herds. American Journal of Veterinary Research 67, 821-828.

van Roermund HJW, Bakker D, Willemsen PTJ et al. (2007) Horizontal transmission of Mycobacterium avium subsp. paratuberculosis in cattle in an experimental setting: Calves can transmit the infection to other calves. Veterinary Microbiology 122, 270-279.

Weber MF (2006) Risk management of paratuberculosis in dairy herds. Irish Veterinary Journal 59, 555-561.

Whitlock RH, Wells SJ, Sweeney RW et al. (2000) ELISA and faecal culture for paratuberculosis (Johne's disease): sensitivity and specificity of each method. Veterinary Microbiology 77, 387-398. 